\title{
Mechanical properties of jennite: a theoretical and experimental study
}

Juhyuk Moon $^{\mathrm{a}, *}$, Seyoon Yoon ${ }^{\mathrm{b}}$, and Paulo J.M. Monteiro ${ }^{\mathrm{c}}$

${ }^{a}$ Civil Engineering Program, Department of Mechanical Engineering, Stony Brook University, New York 11794, USA

${ }^{\mathrm{b}}$ School of Engineering, Kings College, University of Aberdeen, Aberdeen AB24 3UE, U.K.

${ }^{c}$ Department of Civil and Environmental Engineering, University of California, Berkeley, California 94720, USA

* Corresponding author

E-mail address: juhyuk.moon@ @stonybrook.edu

Phone number: +1-631-632-9222

Postal address: 250 Heavy Engineering, Civil Program in Mechanical Engineering, Stony Brook University, Stony Brook NY 11794

\begin{abstract}
The objective of this study is to determine the mechanical properties of jennite. To date, several hypotheses have been proposed to predict the structural properties of jennite. For the first time as reported herein, the isothermal bulk modulus of jennite was measured experimentally. Synchrotron-based high-pressure x-ray diffraction experiments were performed to observe the variation of lattice parameters under pressure. First-principles calculations were applied to compare with the experimental results and predict additional structural properties. Accurately measured isothermal bulk modulus herein $\left(\mathrm{K}_{0}=64(2)\right.$ GPa) and the statistical assessment on experimental and theoretical results suggest reliable mechanical properties of shear and Young's modulus, Poisson's ratio, and elastic tensor coefficients. Determination of these fundamental structural properties are the first step toward greater understanding of calciumsilicate-hydrate, as well as provide a sound foundation for forthcoming atomic level simulations.
\end{abstract}


Keyword: Calcium-Silicate-Hydrate (C-S-H) (B), X-Ray Diffraction (B), Elastic Moduli (C), Elastic Properties (C), Modeling (E)

\section{Introduction}

Cement paste is a porous and hierarchical material consisting of various levels of structures across different length scales [1]. Each scale has a different characteristic size and accordingly different structural properties, which make concrete a highly complex material. The main components of cement paste are poorly crystalline calcium-silicate-hydrate $(\mathrm{C}-\mathrm{S}-\mathrm{H}$ gel) and crystalline phases of portlandite, ettringite, and $\mathrm{Al}_{2} \mathrm{O}_{3}-\mathrm{Fe}_{2} \mathrm{O}_{3}$-mono (AFm) phases, such as monosulfate and monocarboaluminate.

Among the different cement hydration products, the C-S-H gel is the most important component in concrete. Not only because it constitutes a large volume of cement paste, but it is responsible for numerous important properties of concrete, including setting, hardening, shrinkage, and creep [1]. The disordered nature and compositional variance make research on C-S-H gel challenging [2-4]. It has been proposed that C-S-H(I) and C-S-H(II) are semi-crystalline versions of crystalline calcium silicate hydrate minerals, tobermorite $14 \AA$, and jennite, respectively [5-7]. Other researchers have postulated that C-S-H gel can be treated as a mixture of tobermorite- and jennite-like structures. The jennite-like structure is proposed as a dominant phase with age $[2-4,8,9]$.

Jennite, $\mathrm{Ca}_{9}\left(\mathrm{Si}_{6} \mathrm{O}_{18}\right)(\mathrm{OH})_{6} \cdot 8 \mathrm{H}_{2} \mathrm{O}$, is a rare natural mineral, usually found in contact with tobermorite $14 \AA$ [10]. It can be synthesized in suspensions of $\mathrm{Ca}(\mathrm{OH})_{2}$ and hydrous silica at $60-100{ }^{\circ} \mathrm{C}$ [11]. An ideal $\mathrm{Ca} / \mathrm{Si}$ ratio is 1.5 , which is higher than that of tobermorite $14 \AA$ ( $\mathrm{Ca} / \mathrm{Si}$ ratio of 1$)$ [12, 13]. The crystal structure of jennite has been determined by x-ray diffraction refinement [14]. The structure of jennite is composed of (1) the tilleyite ribbons of edge-sharing calcium octahedral; (2) the Si-O dreierketten chains running along [010]; and (3) the additional calcium octahedral sitting between the chains [14]. Upon dehydration at $70-90{ }^{\circ} \mathrm{C}$, water molecules are lost, which alters the atomic environment of interlayer $\mathrm{Ca}$. To be a stable configuration, the structure shrinks in the $c$ direction, leading to metajennite, $\mathrm{Ca}_{9}\left[\mathrm{Si}_{6} \mathrm{O}_{16}(\mathrm{OH})_{2}\right](\mathrm{OH})_{8} \cdot 2 \mathrm{H}_{2} \mathrm{O}$. Above $350{ }^{\circ} \mathrm{C}$, the $\mathrm{Ca}-\mathrm{OH}$ groups of metajennite are eliminated, forming an amorphous phase, which finally transforms to wollastonite and larnite $(\beta$ $\mathrm{Ca}_{2} \mathrm{SiO}_{4}$ ) above $800{ }^{\circ} \mathrm{C}$ [15]. 
With increasing interests in mineral analogs of $\mathrm{C}-\mathrm{S}-\mathrm{H}$, fundamental structural properties are undoubtedly an important part for the understanding of mechanical characteristics of C-S-H. Using firstprinciples calculation [16, 17] and molecular dynamics simulation [18], several studies have been made to predict mechanical properties of tobermorite and jennite. Along with simulation studies, the bulk moduli of tobermorite crystals have been experimentally measured $[19,20]$. For the very first time, the study reported herein measures experimentally the isothermal bulk modulus of jennite. The results report a synchrotron-based x-ray diffraction experiment that gives reliable pressure-volume data for jennite crystal. As a benchmark to the experimental data, first-principles calculations using different exchangecorrelational functionals are performed to support the experimental results. Lastly, statistical assessment on the experimental and theoretical results is discussed.

The remainder of the present study is organized as follows. Experimental procedure and results are given in Section 2. Section 3 focuses on the application of first-principles calculations. The combination of experiment and simulation allows systematic comparison with previous simulation data, and a comprehensive understanding of the structural mechanism is discussed in Section 4 . Section 5 summarizes the major conclusions achieved in this study.

\section{High pressure x-ray experiments}

Jennite crystals (see Fig. 1) from Zeilberg, Bavaria, Germany were obtained for the experiments. Ambient-condition identification was confirmed by laboratory scale x-ray diffraction, and high pressure X-ray diffraction experiments (HPXRD) were carried out at beamline 12.2.2 of the Advanced Light Source [21], using a synchrotron monochromatic x-ray source. Experimental details can be found in previous publications [22-24]. The pressure-transmitting medium consisted of a 4:1 mixture of methanol:ethanol used in the first run and silicone oil for the second run. For the alcohol mixture, an xray wavelength of $\lambda=0.6199 \AA$ and sample-to-detector distance of $221.4 \mathrm{~mm}$ were selected. For the silicone oil, different setting of $\lambda=0.4959 \AA$ and $387.5 \mathrm{~mm}$ was chosen. Ambient and high-pressure $\mathrm{x}$ ray diffraction patterns of jennite are shown in Figs. 2 and 3, measured in the alcohol mixture and silicone oil, respectively. The position and relative intensities of ambient x-ray reflections agree well with the data obtained in Ref. [22]. Unindexed peaks of $4.7^{\circ}$ and $9.4^{\circ}$ in Fig. 2 and the observed hump in Fig. 3 might have resulted from the diamond anvil cell or impurities in the pressure transmitting media. Almost identical results from different media indicate that these did not affect hydrostatic state on the jennite crystals. 
Accurate peak positions were determined by a pseudo-Voight function implemented in XFit software [25]. The peak positions are summarized in Tables 1 and 2. Due to its low symmetry, it is quite challenging to refine lattice parameters of jennite. The mathematical relation between $d$-spacing and Miller indices in triclinic symmetry is,

$$
\begin{aligned}
& \frac{1}{d^{2}}=\frac{1}{V^{2}}\left(S_{11} h^{2}+S_{22} k^{2}+S_{33} l^{2}+2 S_{12} h k+2 S_{23} k l+2 S_{13} h l\right) \\
& \text { where } S_{11}=b^{2} c^{2} \sin ^{2} \alpha \\
& \qquad \begin{aligned}
S_{22} & =a^{2} c^{2} \sin ^{2} \beta \\
S_{33} & =a^{2} b^{2} \sin ^{2} \gamma \\
S_{12} & =a b c^{2}(\cos \alpha \cos \beta-\cos \gamma) \\
S_{23} & =a^{2} b c(\cos \beta \cos \gamma-\cos \alpha) \\
S_{13} & =a b^{2} c(\cos \gamma \cos \alpha-\cos \beta)
\end{aligned}
\end{aligned}
$$

Nonlinear regression using the Newton-Raphson method and normal distribution (also known as Gaussian function) [26] was used to refine the lattice parameters of jennite. X-ray diffraction peaks given in Tables 1 and 2 were selected for the nonlinear regression method. Given the $d$-spacing and hkl information, lattice parameters of $a, b, c, \alpha, \beta$, and $\gamma$ were successfully refined by iteratively solving Eqn. (1). Pressure above $2.9 \mathrm{GPa}$ resulted in x-ray diffraction peaks that were broader and overlapped considerably; therefore, refinement of unit cell was not successful, regardless of the type of pressuretransmitting media. The refined lattice parameters using the alcohol mixture versus silicone oil are summarized in Table 3. Note that standard deviations in the unit cell are dependent on the number and selection of peaks used for the refinement. Refinement in the case of ambient pressure and silicone oil yielded larger deviations compared to the refinement in the case of the alcohol mixture.

The refined ambient lattice parameters agree well with the results of Bonaccorsi et al. [14], with less than $1 \%$ error range (Table 3). Nevertheless, the existence of disorder in the jennite crystal structure $[14,27,28]$ and the anomalous hump from an external source made it difficult to apply the Rietveld refinement method to the experimental data. Application of the nonlinear regression method, however, 
gave an acceptable error range in lattice parameters considering the complexity of crystal structure of jennite. The variation of lattice parameters under pressure is shown in Figs. 4 and 5. As stated in early this section, it shows almost identical results in both media.

The pressure normalized volume data were fitted by a second- and third- order finite strain equation of state (i.e., the Murnaghan and Birch-Murnaghan equation of state) [29]. Below is the BirchMurnaghan equation of state (BM EoS).

$$
P(V)=\frac{3}{2} K_{0}\left\{\left(\frac{V}{V_{0}}\right)^{-\frac{7}{3}}-\left(\frac{V}{V_{0}}\right)^{-\frac{5}{3}}\right\}\left\lfloor 1+\frac{3}{4}\left(K_{0}^{\prime}-4\right)\left\{\left(\frac{V}{V_{0}}\right)^{-\frac{2}{3}}-1\right\}\right\rfloor \quad \text { Eqn. (2) }
$$

where $V$ is volume of the unit cell, $V_{0}$ is initial volume of the unit cell at ambient pressure, $P$ is the pressure applied to a material, $K_{0}$ is the bulk modulus at zero pressure, and $K_{0}^{\prime}$ is the derivative of the bulk modulus at zero pressure. By defining the normalized pressure, $F$ and the Eulerian strain, $f$, the third order BM EoS is reorganized into the linear form:

$$
\begin{array}{lc}
F(f)=K_{0}-1.5 K_{0}\left(4-K_{0}^{\prime}\right) f & \text { Eqn. (3) } \\
\text { where } f=\frac{1}{2}\left\lfloor\left(\frac{V}{V_{0}}\right)^{-2 / 3}-1\right\rfloor & \text { Eqn. (4) }
\end{array}
$$

The weighted linear least-squares fitting in a plot of $F$ versus $f$ [Fig. 5(b)] gives the bulk modulus $K_{0}$ and its derivative $K_{0}^{\prime}$ from $y$-intercept and slope of graph, respectively. In the case of the alcohol mixture, the $3^{\text {rd }} \mathrm{BM}$ EoS determines a bulk modulus of 45(5) GPa and a derivative of 42.3, with an accuracy of fit of $\mathrm{R}^{2}=0.997$. In addition, the $2^{\text {nd }} \mathrm{BM}$ EoS fit gives a bulk modulus of $68(5) \mathrm{GPa}$ with $\mathrm{R}^{2}$ $=0.970$. In the case of the silicone oil, the $3^{\text {rd }} \mathrm{BM}$ EoS determines a bulk modulus of 61(4) $\mathrm{GPa}$ and a derivative of 9.9 with an accuracy of fit of $\mathrm{R}^{2}=0.964$. Finally, the $2^{\text {nd }} \mathrm{BM}$ EoS fit gives a bulk modulus of 64(2) GPa with $\mathrm{R}^{2}=0.956$. The graphical fitting result is shown in Figs. 5(a) and (b).

\section{First-principles calculations}

This section discusses the first-principles calculation of jennite. The initial crystal structure for simulation has triclinic symmetry $\left(a=10.576 \AA, b=7.265 \AA, c=10.931 \AA, \alpha=101.3^{\circ}, \beta=96.98^{\circ}\right.$, and $\gamma$ 
$=109.65^{\circ}$ ) as determined by Ref, [14]. The missing hydrogen atoms from x-ray diffraction experiment were recently proposed through density functional theory (DFT) calculation by Churakov [30]. This hydrogen bonding scheme was selected as an initial configuration for investigating structural properties of jennite. All computations were performed on Linux clusters in the Molecular Graphics and Computation Facility at the University of California, Berkeley. The DFT calculations were performed using LocalDensity-Approximation (LDA) and Perdew-Burke-Ernzerhof (PBE) Generalized-GradientApproximation (GGA) [31] exchange-correlation functionals and plane wave techniques implemented in the Quantum ESPRESSO distribution [32]. Ultrasoft type exchange-correlation functionals [33] were used with a plane-wave energy cut-off of $1900 \mathrm{eV}$ and $4 \times 2 \times 2 k$-points sampling [34]. The reference valence configurations and core radii for GGA and LDA functionals were chosen as $3 \mathrm{~s}^{2}, 3 \mathrm{p}^{6}, 4 \mathrm{~s}^{2}, \mathrm{r}_{-} \mathrm{c}=$ $1.2 \AA$ for $\mathrm{Ca}, 3 \mathrm{~s}^{2}, 3 \mathrm{p}^{2}, \mathrm{r} \_\mathrm{c}=1.2 \AA$ for $\mathrm{Si}, 2 \mathrm{~s}^{2}, 2 \mathrm{p}^{4}, \mathrm{r} \_\mathrm{c}=0.8 \AA$ for $\mathrm{O}$, and $1 \mathrm{~s}^{1}$, for $\mathrm{H}$.

Before calculating the structural properties, geometrical optimizations were performed at zero pressure. Lattice parameters and fractional atomic positions were iteratively changed until computed forces were smaller than $10^{-4} \mathrm{eV} / \AA$, and total energy difference was less than $10^{-6} \mathrm{eV}$. The residual stress components of the optimized structure were less than 0.1 kbar. The geometrically optimized crystal structure is shown in Fig. 6. Likewise, equilibrium structures at different pressures (-1.0, 1.0, 2.0, 3.0, 4.0, and 5.0 GPa) were obtained using damped variable cell shape molecular dynamics [35]. Resulting lattice parameters at different pressures using LDA and GGA are presented in Table 4 and Figs. 4 and 7.

Elastic coefficients of jennite were calculated based on a stress-strain relation. For infinitesimal strains this relation is linear.

$$
\sigma_{i}=\sum_{j=1}^{6} C_{i j} \varepsilon_{j}
$$

Individual strains were applied to the equilibrium structure (i.e., structural optimization at $0 \mathrm{GPa}$ ). Next, internal structural degrees of freedom were re-optimized. Residual stress components for the reoptimized structures were less than $0.1 \mathrm{kbar}$. Twenty-one independent elastic coefficients for jennite were calculated based on two different crystallographic settings of $(\vec{Z}\|\vec{c}, \vec{Y}\| \vec{c} \times \vec{a}$, and $\vec{X} \| \vec{Y} \times \vec{Z})$ and ( $\vec{X}\|\vec{a}, \vec{Z}\| \vec{a} \times \vec{b}$, and $\vec{Y} \| \vec{Z} \times \vec{X}$ ). Note that the $\vec{Z} \| \vec{c}$ setting is conventional for triclinic system [36, 37], while the $\vec{X} \| \vec{a}$ setting for systematical comparison of the elastic constants from different crystal structure. Applied Lagrangian strains in Cartesian coordinates result in: 


$$
\begin{aligned}
& \varepsilon_{1}=\left(\begin{array}{lll}
\delta & 0 & 0 \\
0 & 0 & 0 \\
0 & 0 & 0
\end{array}\right), \\
& \varepsilon_{2}=\left(\begin{array}{lll}
0 & 0 & 0 \\
0 & \delta & 0 \\
0 & 0 & 0
\end{array}\right), \\
& \varepsilon_{3}=\left(\begin{array}{lll}
0 & 0 & 0 \\
0 & 0 & 0 \\
0 & 0 & \delta
\end{array}\right), \\
& \varepsilon_{4}=\left(\begin{array}{lll}
0 & 0 & 0 \\
0 & 0 & \delta / 2 \\
0 & \delta / 2 & 0
\end{array}\right), \\
& \varepsilon_{5}=\left(\begin{array}{ccc}
0 & 0 & \delta / 2 \\
0 & 0 & 0 \\
\delta / 2 & 0 & 0
\end{array}\right), \\
& \varepsilon_{6}=\left(\begin{array}{ccc}
0 & \delta / 2 & 0 \\
\delta / 2 & 0 & 0 \\
0 & 0 & 0
\end{array}\right),
\end{aligned}
$$

where the indices are given in Voigt notation. Sufficiently small strains of $\delta= \pm 0.5 \%$ were applied, and elastic coefficients were obtained by averaging stresses resulting from positive and negative strains. Table 5 summarizes the computed elastic coefficients of jennite from LDA and GGA exchangecorrelation functionals.

Adiabatic mechanical constants can be derived from the computed elastic constants [38, 39]. The bound values were computed using elastic coefficients, $C$, determined from Eqn. (5). From the computed $K_{\mathrm{RVH}}$ and $G_{\mathrm{RVH}}$, the Young's modulus $(E)$ and Poisson ratio $(\eta)$ were also calculated. The RVH mechanical properties were computed and are summarized in Table 5. The isothermal bulk modulus was obtained by fitting Eqn. (2) to the theoretically computed pressure-volume data (Table 4). As discussed 
in Section 2, this fitting gives the isothermal bulk modulus at zero pressure and its first derivative. The results are given in Table 6.

The Young's modulus for uniaxial compression along arbitrary directions was computed. The general definition of the directional Young's modulus in terms of unit vectors, $\hat{n}$, along the compression axis is:

$$
E_{a n i}=\frac{1}{\hat{n}^{T} \cdot\left[C^{-1}:(\hat{n} \cdot \hat{n})\right] \cdot \hat{n}}
$$

Calculated magnitudes of the LDA and GGA Young's modulus are represented in colors on the surface of the sphere (Fig. 8). The $X, Y$, and $Z$ directions are defined in $(\vec{X}\|\vec{a}, \vec{Z}\| \vec{a} \times \vec{b}$, and $\vec{Y} \| \vec{Z} \times \vec{X})$ setting. The large structural anisotropy of jennite is evident in this figure. Although the relaxed lattice volume from LDA $\left(811.99 \AA^{3}\right)$ is $6 \%$ larger than that from GGA $\left(765.22 \AA^{3}\right)$, it shows a similar range of the Young's modulus and a tendency of variation depending on the direction.

\section{Discussion}

High-pressure x-ray diffraction allows direct measurement of isothermal bulk modulus. Since it corresponds to the first derivative of Helmholtz free energy with respect to volume, it provides useful information on relative thermo-dynamical stability of a material [40]. The previous study on AFm phases shows pressure-induced dehydration at low pressures (below $2 \mathrm{GPa}$ ) [22]. In addition, sudden phase transition of tobermorite at high pressure (over $10 \mathrm{GPa}$ ) is proposed by DFT simulation [41]. In the case of jennite, no pressure-induced changes were observed, regardless of the type of pressure-transmitting medium; however, the diffraction peaks were overlapped significantly over $2.9 \mathrm{GPa}$. Although the refinement of lattice parameters was not successful at this pressure level, no phase transitions were predicted by DFT simulations. The variation of lattice parameters is shown in Fig. 4. Similar to common layered minerals, the softest lattice parameter is $c$ lattice parameter, which is almost perpendicular to the principal layer.

Table 4 summarizes calculated lattice parameters at different pressures. At zero pressure, both exchange-correlation functionals overestimate a unit-cell volume. The LDA overestimates the initial volume by $6 \%\left(811.99 \AA^{3}\right.$ versus $\left.759.5 \AA^{3}\right)$, but GGA precisely predicts the value within $1 \%$ difference (765.22 $\AA^{3}$ vs. $759.5 \AA^{3}$ ) [14]. As shown in Fig. 4(a), the overestimation in LDA is mainly due to the 
predicted length lattice parameters. Table 6 presents the mechanical properties of jennite obtained from high-pressure x-ray diffraction and first-principles calculations. The large $K_{0}^{\prime}$ value in $3^{\text {rd }}$ order EoS is due to the small number of data points [Fig. 5(b)]. It is not enough to take into consideration of nonlinear effect of a material under high pressure [22-24]; therefore, the fitting result of $2^{\text {nd }}$ order EoS is chosen for comparison with the simulation results. While GGA $\left(K_{0}=54.2(8) \mathrm{GPa}\right)$ underestimates the experimental results (64-68 GPa), LDA calculation yields a closer value of $K_{0}=63(1) \mathrm{GPa}$. This can be confirmed by the pressure-volume data shown in Fig. 7. In the case of oxide mineral, the LDA calculation usually tends to overestimate the mechanical properties while GGA underestimates them [4244]. In this study, LDA overestimated a unit cell volume, but it shows better performance on pressurevolume behavior (i.e., represented as an isothermal bulk modulus) as shown in Fig. 7. Therefore, it can be suggested that the mechanical properties (Table 6), elastic tensor coefficients (Table 5) and anisotropic feature (Fig. 9) from the LDA calculations are fairly accurate.

In addition, a statistical assessment has been performed on experimental and theoretical results. Residuals are defined as the difference between an experimental (observed) value and simulated (predicted) one. The Quantile-Quantile (or Q-Q plot) evaluation, where the cumulative probability of the residuals is compared to that of the theoretical normal distribution, was applied to evaluate the normality of the residuals. In the Q-Q plot, if the residuals lie closely on a straight line, they follow the normal distribution. The residuals of lengths and angles in lattice parameters obtained from LDA and GGA are shown in Fig. 9. Note that the residuals obtained from GGA are characterized by heavy tails in the Q-Q plots, whereas the residuals from LDA show better linearity in both length and angle of the lattice parameters. For quantification of this visual observation, the mean squared residuals (MSR) were computed from the residuals. As shown in Table 7, lower MSR values were obtained from LDA, while GGA showed higher MSR values. Therefore, these Q-Q plots and MSRs suggest that LDA functionals have less biased residuals than GGA functionals do.

It is important to note the lack of a van der Walls ( VdW) interaction in current simulation study may introduce errors. The DFT may not yield accurate VdW dispersion forces due to its long-range electrostatic characteristic. In addition, there is a difference between the results obtained from firstprinciples calculations versus molecular dynamic (MD) simulations (Fig. 7 and Tables 5 and 6). While the accuracy of MD simulation is highly dependent on empirical force fields, first-principles calculations do not depend on anything except the exchange-correlation functional. In this study, the LDA and GGA calculations predict isothermal bulk modulus (from $2^{\text {nd }}$ order EoS) as 63(1) GPa and 54.2(8) GPa, respectively. The GGA calculation obtained in Ref. [16] predicted an RVH bulk modulus as $32 \mathrm{GPa}$, 
which is much smaller than the results from both simulation reported herein and high-pressure $\mathrm{x}$-ray experiment $\left(\mathrm{K}_{\mathrm{RVH}}=60-63 \mathrm{GPa}\right.$ and $\mathrm{K}_{0}=64-68 \mathrm{GPa}$, respectively). Similarly, there is a significant difference of elastic constants, especially for $C_{22}$ and $C_{33}$ (Table 5). The previous GGA calculation used a setting of $(\vec{X}\|\vec{a}, \vec{Z}\| \vec{a} \times \vec{b}$, and $\vec{Y} \| \vec{Z} \times \vec{X}$ ). In this study, twenty-one independent elastic coefficients for jennite were calculated based on two different crystallographic settings of ( $\vec{Z}\|\vec{c}, \vec{Y}\| \vec{c} \times \vec{a}$, and $\vec{X} \| \vec{Y} \times \vec{Z})$ and $(\vec{X}\|\vec{a}, \vec{Z}\| \vec{a} \times \vec{b}$, and $\vec{Y} \| \vec{Z} \times \vec{X})$; thus, direct comparison of each elastic constant is possible. The lower values of $C_{22}$ and $C_{33}$ in previous calculations result in the lower RVH bulk modulus (Table 5) [16]. However, considering electron densities and layer structural characteristics (Figs. 6 and 8), $C_{22}$ might be the least compressible direction in the system compared to $C_{11}$ and $\mathrm{C}_{33}$. (The $\mathrm{C}_{22}$ indicates compressibility of the parallel direction to principal layer.) This tendency is also confirmed by a recent DFT study on jennite using LDA functionals [17], indicating that $C_{22}$ is the stiffest constant (Table $5)$.

Another possible error in [16] may be due to unreasonable assumptions of the initial structure, especially for hydrogen positions in water molecules and hydroxides. Their positions had not been resolved by previous x-ray diffraction methods [14]; reliable positions have been recently suggested by Ref. [30]. In the case of a layer structure containing water and hydroxides, the hydrogen bonding network plays a key role in the mechanical properties [19, 22, 23, 45]. Therefore, assuming inaccurate initial positions of hydrogens can likely induce the system to fall in local potential minima. In addition, the convergence criteria are the important parameters for successful first-principles calculation. Limited criteria can save computational costs, but it can yield inaccurate simulation results. Although further study is necessary to verify this discrepancy, it is suggested that high-pressure x-ray diffraction can be an ideal experimental technique to evaluate the performance of various simulation methods.

\section{Conclusion}

The complexity of small-scale concrete research stems from the lack of experimental data at the fundamental level. This study focuses on mechanical properties of materials that determine the overall performance of concrete. The mechanical properties of jennite were experimentally and theoretically investigated. Under pressure, the variations of $a$ and $b$ lattice parameters were small compared to that of 
the $c$ lattice parameter. In addition, the directional Young's moduli greatly depend on the direction in compression. This confirms the layered structural characteristics of jennite.

The good agreement between experimental and theoretical pressure-volume behaviors (i.e., represented as isothermal bulk modulus) suggests a noble combined methodology to obtain accurate static properties. While GGA exchange-correlation functionals predict accurate lattice parameters, it underestimates the bulk modulus. On the other hand, LDA functionals overestimate the experimental lattice parameters and a unit cell volume but its prediction on bulk modulus is closer to experimental data. This tendency was also observed from previous simulation research on jennite. From the systematic comparison between the experiment and simulation, the reliable mechanical properties of bulk, shear, and Young's modulus, Poisson's ratio, and elastic constants were proposed. High-pressure x-ray diffraction techniques can validate the performance of different simulation methods. In first-principles calculations, setting high convergence criteria and reasonable initial atomic configuration can be important factors to avoid any local potential minima.

The systematic measurements of the modulus of jennite can provide insights on the structural composition of cement paste at a small scale and on the deformation properties of C-S-H gel. Furthermore, the development of accurate empirical potentials for C-S-H is an important task to study nano-particle clusters of $\mathrm{C}-\mathrm{S}-\mathrm{H}$, as well as for performing simulations of a large system of materials at low computational costs. The fundamental mechanical properties reported herein will be a key basis for forthcoming molecular-level simulations of cement and concrete.

\section{Acknowledgement}

The research reported herein was carried out at the Advanced Light Source, which is supported by the Director, Office of Science, Office of Basic Energy Sciences, of the U.S. Department of Energy under Contract No. DE-AC02-05CH11231 and at the Center for Functional Nanomaterials, Brookhaven National Laboratory, which is supported by the U.S. Department of Energy, Office of Basic Sciences, under contract no. DE-AC02-98CH10886. The U.C. Berkeley Molecular Graphics and Computation Facility is supported by under Grant Nu. NSF/CHE-0840505.

\section{References}


[1] P.K. Mehta, P.J.M. Monteiro, CONCRETE-Microstructure, Properties, and Materials, 3 (2006).

[2] I. Richardson, Tobermorite/jennite-and tobermorite/calcium hydroxide-based models for the structure of CSH: applicability to hardened pastes of tricalcium silicate, $\beta$-dicalcium silicate, Portland cement, and blends of Portland cement with blast-furnace slag, metakaolin, or silica fume, Cement and Concrete Research, 34 (2004) 1733-1777.

[3] I. Richardson, The calcium silicate hydrates, Cement and Concrete Research, 38 (2008) 137-158. [4] A.J. Allen, J.J. Thomas, H.M. Jennings, Composition and density of nanoscale calcium-silicatehydrate in cement, Nature materials, 6 (2007) 311-316.

[5] H. Taylor, J. Howison, Relationships between calcium silicates and clay minerals, Clay Minerals Bull, 3 (1956) 98-111.

[6] H. Taylor, The calcium silicate hydrates, The chemistry of cements, 1 (1964) 162-232.

[7] J. Gard, H. Taylor, Calcium silicate hydrate (II)(“CSH (II)"), Cement and Concrete Research, 6 (1976) 667-677.

[8] H.M. Jennings, A model for the microstructure of calcium silicate hydrate in cement paste, Cement and Concrete Research, 30 (2000) 101-116.

[9] H.M. Jennings, J.W. Bullard, J.J. Thomas, J.E. Andrade, J.J. Chen, G.W. Scherer, Characterization and modeling of pores and surfaces in cement paste: correlations to processing and properties, Journal of Advanced Concrete Technology, 6 (2008) 5-29.

[10] D.L. Carpenter, Whistler studies of the plasmapause in the magnetosphere 1. Temporal variations in the position of the knee and some evidence on plasma motions near the knee, Journal of Geophysical Research, 71 (1966) 693-709.

[11] N. Hara, N. Inoue, Formation of jennite from fumed silica, Cement and Concrete Research, 10 (1980) 677-682.

[12] N. Hara, N. Inoue, H. Noma, Formation of Jennite and Tobermorite and their Relationship with CS-H Gel in Hydrated Cement Paste, in: Materials Science of Concrete-The Sidney Diamond Symposium Special Volume Proceedings of the Professor Sidney Diamond Symposium, Honolulu, Hawaii Edited by M Cohen, S Mindess, and J Skalny, The American Ceramic Society, Westerville, OH, 1998, pp. 71-80.

[13] E. Bonaccorsi, S. Merlino, A.R. Kampf, The crystal structure of tobermorite 14 A (plombierite), a CS-H phase, Journal of the American Ceramic Society, 88 (2005) 505-512.

[14] E. Bonaccorsi, S. Merlino, H. Taylor, The crystal structure of jennite, $\mathrm{Ca}_{9} \mathrm{Si}_{6} \mathrm{O}_{18}(\mathrm{OH})_{6} \cdot 8 \mathrm{H}_{2} \mathrm{O}$, Cement and Concrete Research, 34 (2004) 1481-1488.

[15] P. Yu, R. Kirkpatrick, Thermal dehydration of tobermorite and jennite, Concrete Science and Engineering, 1 (1999) 185-191.

[16] R. Shahsavari, M.J. Buehler, R.J.M. Pellenq, F.J. Ulm, First-Principles Study of Elastic Constants and Interlayer Interactions of Complex Hydrated Oxides: Case Study of Tobermorite and Jennite, Journal of the American Ceramic Society, 92 (2009) 2323-2330.

[17] C. Dharmawardhana, A. Misra, S. Aryal, P. Rulis, W. Ching, Role of interatomic bonding in the mechanical anisotropy and interlayer cohesion of CSH crystals, Cement and Concrete Research, 52 (2013) 123-130.

[18] H. Manzano, J. Dolado, A. Guerrero, A. Ayuela, Mechanical properties of crystalline calcium-silicate-hydrates: comparison with cementitious C-S-H gels, physica status solidi (a), 204 (2007) 1775-1780.

[19] M.D. Jackson, J. Moon, E. Gotti, R. Taylor, S.R. Chae, M. Kunz, A.H. Emwas, C. Meral, P. Guttmann, P. Levitz, Material and Elastic Properties of Al-Tobermorite in Ancient Roman Seawater Concrete, Journal of the American Ceramic Society, (2013).

[20] J.E. Oh, S.M. Clark, H.-R. Wenk, P.J. Monteiro, Experimental determination of bulk modulus of $14 \AA ̊$ tobermorite using high pressure synchrotron X-ray diffraction, Cement and Concrete Research, 42 (2012) 397-403. 
[21] M. Kunz, A.A. MacDowell, W.A. Caldwell, D. Cambie, R.S. Celestre, E.E. Domning, R.M. Duarte, A.E. Gleason, J.M. Glossinger, N. Kelez, A beamline for high-pressure studies at the Advanced Light Source with a superconducting bending magnet as the source, Journal of synchrotron radiation, 12 (2005) 650-658.

[22] J.-h. Moon, J.E. Oh, M. Balonis, F.P. Glasser, S.M. Clark, P.J. Monteiro, Pressure induced reactions amongst calcium aluminate hydrate phases, Cement and Concrete Research, 41 (2011) 571-578.

[23] J. Moon, J.E. Oh, M. Balonis, F.P. Glasser, S.M. Clark, P.J. Monteiro, High pressure study of low compressibility tetracalcium aluminum carbonate hydrates $3 \mathrm{CaO} \cdot \mathrm{Al}_{2} \mathrm{O}_{3} \cdot \mathrm{CaCO}_{3} \cdot 11 \mathrm{H}_{2} \mathrm{O}$, Cement and Concrete Research, 42 (2012) 105-110.

[24] J. Moon, S. Yoon, R.M. Wentzcovitch, S.M. Clark, P.J. Monteiro, Elastic Properties of Tricalcium Aluminate from High-Pressure Experiments and First-Principles Calculations, Journal of the American Ceramic Society, 95 (2012) 2972-2978.

[25] R. Cheary, A. Coelho, Programs xfit and fourya, Deposited in CCP14 Powder Diffraction Library, Engineering and Physical Sciences Research Council, Daresbury Laboratory, Warrington, England, (1996).

[26] J. Neter, M.H. Kutner, C.J. Nachtsheim, W. Wasseman, Applied linear statistical models, (1996).

[27] J. Gard, H. Taylor, G. Cliff, G. Lorimer, A reexamination of jennite, American Mineralogist, 62 (1977) 365-368.

[28] D. Viehland, L.J. Yuan, Z. Xu, X.D. Cong, R.J. Kirkpatrick, Structural studies of jennite and $1.4 \mathrm{~nm}$ tobermorite: Disordered layering along the [100] of jennite, Journal of the American Ceramic Society, 80 (1997) 3021-3028.

[29] F. Birch, Finite strain isotherm and velocities for single-crystal and polycrystalline $\mathrm{NaCl}$ at high pressures and 300 K, Journal of Geophysical Research, 83 (1978) 1257-1268.

[30] S.V. Churakov, Hydrogen bond connectivity in jennite from ab initio simulations, Cement and Concrete Research, 38 (2008) 1359-1364.

[31] J.P. Perdew, K. Burke, M. Ernzerhof, Generalized Gradient Approximation Made Simple, Physical Review Letters, 77 (1996) 3865-3868.

[32] G. Paolo, et al., QUANTUM ESPRESSO: a modular and open-source software project for quantum simulations of materials, Journal of Physics: Condensed Matter, 21 (2009) 395502.

[33] D. Vanderbilt, Soft self-consistent pseudopotentials in a generalized eigenvalue formalism, Physical Review B, 41 (1990) 7892.

[34] H.J. Monkhorst, J.D. Pack, Special points for Brillouin-zone integrations, Physical Review B, 13 (1976) 5188-5192.

[35] R.M. Wentzcovitch, Invariant molecular-dynamics approach to structural phase transitions, Physical Review B, 44 (1991) 2358.

[36] S. Matthies, H.-R. Wenk, Transformations for monoclinic crystal symmetry in texture analysis, Journal of Applied Crystallography, 42 (2009) 564-571.

[37] B. Militzer, H.-R. Wenk, S. Stackhouse, L. Stixrude, First-principles calculation of the elastic moduli of sheet silicates and their application to shale anisotropy, American Mineralogist, 96 (2011) 125-137.

[38] R. Hill, The Elastic Behaviour of a Crystalline Aggregate, Proceedings of the Physical Society Section A, 65 (1952).

[39] D. J.P. Watt, G.F., and O'Connell, R.J., The elastic properties of composite materials, Reviews of Geophysics, 14 (1976).

[40] J. Moon, S. Yoon, R.M. Wentzcovitch, P.J. Monteiro, First-principles elasticity of monocarboaluminate hydrates, American Mineralogist, 99 (2014) 1360-1368.

[41] A. Zaoui, Insight into elastic behavior of calcium silicate hydrated oxide $(\mathrm{C}-\mathrm{S}-\mathrm{H})$ under pressure and composition effect, Cement and concrete research, 42 (2012) 306-312.

[42] R.M. Wentzcovitch, Z. Wu, P. Carrier, First principles quasiharmonic thermoelasticity of mantle minerals, Reviews in Mineralogy and Geochemistry, 71 (2010) 99-128. 
[43] R. Wentzcovitch, L. Stixrude, J.J. Rosso, Theoretical and computational methods in mineral physics: geophysical applications, Mineralogical Society of America, 2010.

[44] B.B. Karki, L. Stixrude, R.M. Wentzcovitch, High-pressure elastic properties of major oxide, Reviews of Geophysics, 39 (2001) 507-534.

[45] H. Manzano, A. Ayuela, A. Telesca, P. Monteiro, J. Dolado, Ettringite Strengthening at High Pressures Induced by the Densification of the Hydrogen Bond Network, The Journal of Physical Chemistry C, 116 (2012) 16138-16143. 
Table 1. Measured peak positions $(\AA)$ of jennite at ambient pressure and under pressure (Methanol:Ethanol = 4:1).

\begin{tabular}{|c|c|c|c|c|c|c|c|c|c|c|}
\hline \multirow[b]{2}{*}{$\mathbf{h}$} & \multirow[b]{2}{*}{$\mathbf{k}$} & \multirow[b]{2}{*}{ I } & \multirow{2}{*}{$\begin{array}{c}\text { [14] } \\
\text { ambient }\end{array}$} & \multicolumn{7}{|c|}{ X-ray diffraction peak positions (Methanol:Ethanol = 4:1) } \\
\hline & & & & ambient & $\begin{array}{c}0.3(1) \\
\mathrm{GPa}\end{array}$ & $\begin{array}{c}0.5(1) \\
\mathrm{GPa}\end{array}$ & $\begin{array}{c}1.3(1) \\
\mathrm{GPa}\end{array}$ & $\begin{array}{c}1.6(1) \\
\mathrm{GPa}\end{array}$ & $\begin{array}{c}2.0(2) \\
\mathrm{GPa}\end{array}$ & $\begin{array}{c}2.6(2) \\
\mathrm{GPa}\end{array}$ \\
\hline 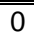 & 0 & 1 & 10.4961 & 10.4835 & 10.4629 & 10.4183 & 10.3529 & 10.3262 & 10.2888 & 10.2193 \\
\hline 1 & 0 & 0 & 9.7528 & 9.7680 & 9.7478 & 9.7324 & 9.7158 & 9.7144 & 9.6994 & 9.6898 \\
\hline 1 & 0 & 1 & 6.5156 & 6.4933 & 5.0321 & 5.0195 & 5.0002 & 4.9959 & 4.9899 & 4.9782 \\
\hline 0 & 0 & 2 & 5.2480 & 5.2291 & - & - & - & - & - & - \\
\hline 1 & 1 & -1 & 4.8438 & 4.8212 & 4.8271 & 4.8374 & 4.8186 & 4.8078 & 4.7934 & 4.7848 \\
\hline 0 & 1 & -2 & 4.7378 & 4.7338 & - & - & - & - & - & - \\
\hline 2 & -1 & -1 & 4.4545 & 4.4619 & 4.4547 & 4.4415 & 4.4314 & 4.4233 & 4.4125 & 4.4083 \\
\hline 1 & 0 & 2 & 4.2737 & 4.2699 & - & - & - & - & - & - \\
\hline 1 & 1 & -2 & 4.1312 & 4.1196 & 4.1198 & 4.1001 & 4.0977 & 4.0917 & 4.0828 & 4.0658 \\
\hline 1 & -1 & 2 & 3.9271 & 3.9224 & - & - & - & - & - & - \\
\hline 0 & 0 & 3 & 3.4987 & 3.4794 & 3.4788 & 3.4650 & 3.4486 & 3.4459 & 3.4421 & 3.4396 \\
\hline 3 & 0 & -1 & 3.3005 & 3.2936 & 3.2938 & 3.2879 & 3.2813 & 3.2779 & 3.2725 & 3.2675 \\
\hline 2 & 0 & 2 & 3.2578 & 3.2508 & - & - & - & - & - & - \\
\hline 1 & -2 & -1 & 3.1847 & 3.1729 & 3.1760 & 3.1687 & 3.1568 & 3.1533 & 3.1420 & 3.1329 \\
\hline 3 & 0 & -2 & 3.0551 & 3.0481 & 3.0502 & 3.0418 & 3.0326 & 3.0294 & 3.0229 & 3.0181 \\
\hline 3 & 0 & 1 & 2.9413 & 2.9340 & 2.9306 & 2.9257 & 2.9181 & 2.9152 & 2.9090 & 2.9034 \\
\hline 3 & -2 & 1 & 2.8451 & 2.8244 & - & - & - & - & - & - \\
\hline 3 & 0 & -3 & 2.6666 & 2.6600 & 2.6578 & 2.6511 & 2.6402 & 2.6373 & 2.6314 & 2.6279 \\
\hline 2 & -2 & 3 & 2.5886 & 2.5930 & - & - & - & - & - & - \\
\hline 0 & 2 & 2 & 2.5255 & 2.5252 & 2.5084 & 2.5019 & 2.4956 & 2.4927 & 2.4904 & 2.4845 \\
\hline 4 & -1 & 2 & 2.2438 & - & 2.2413 & 2.2356 & 2.2282 & 2.2286 & 2.2247 & 2.2210 \\
\hline 2 & 2 & 1 & 2.1780 & - & 2.1676 & 2.1638 & 2.1552 & 2.1525 & 2.1476 & 2.1441 \\
\hline 5 & -2 & 0 & 2.0433 & - & 2.0425 & 2.0382 & 2.0338 & 2.0330 & 2.0293 & 2.0259 \\
\hline 5 & -2 & 1 & 1.9848 & - & 1.9845 & 1.9836 & 1.9774 & 1.9765 & 1.9737 & 1.9730 \\
\hline 2 & -4 & 1 & 1.8154 & 1.8100 & 1.8113 & 1.8101 & 1.8048 & 1.8010 & 1.7999 & 1.7966 \\
\hline
\end{tabular}


Table 2. Measured peak positions ( $\mathrm{A})$ of jennite at ambient pressure and under pressure (Silicone oil).

\begin{tabular}{|c|c|c|c|c|c|c|c|c|c|}
\hline \multirow[b]{2}{*}{ h } & \multirow[b]{2}{*}{$\mathbf{k}$} & \multirow[b]{2}{*}{$\mathbf{I}$} & \multirow{2}{*}{$\begin{array}{c}\text { [14] } \\
\text { ambient }\end{array}$} & \multicolumn{6}{|c|}{ X-ray diffraction peak positions (Silicone oil) } \\
\hline & & & & ambient & $\begin{array}{c}0.1(1) \\
\mathrm{GPa}\end{array}$ & $\begin{array}{c}0.5(1) \\
\mathrm{GPa}\end{array}$ & $\begin{array}{c}0.9(1) \\
\mathrm{GPa}\end{array}$ & $\begin{array}{r}1.3(1) \\
\mathrm{GPa}\end{array}$ & $\begin{array}{c}2.0(1) \\
\mathrm{GPa}\end{array}$ \\
\hline 0 & 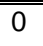 & 1 & 10.4961 & 10.4835 & 10.4610 & 10.4020 & 10.3514 & 10.3261 & 10.2587 \\
\hline 1 & 0 & 0 & 9.7528 & 9.7680 & 9.7281 & 9.7161 & 9.7081 & - & 9.7041 \\
\hline 1 & 0 & 1 & 6.5156 & 6.4933 & 6.5030 & 6.4921 & 6.4565 & 6.4483 & 6.3991 \\
\hline 0 & 0 & 2 & 5.2480 & 5.2291 & 5.2245 & 5.2020 & 5.1846 & 5.1672 & 5.1398 \\
\hline 1 & 1 & -1 & 4.8438 & 4.8212 & 4.8212 & 4.8170 & 4.8058 & 4.8030 & 4.6881 \\
\hline 0 & 1 & -2 & 4.7378 & 4.7338 & 4.7326 & 4.7133 & 4.6937 & 4.6783 & - \\
\hline 2 & -1 & -1 & 4.4545 & 4.4619 & 4.4498 & 4.4476 & 4.4243 & 4.4215 & - \\
\hline 1 & 0 & 2 & 4.2737 & 4.2699 & 4.2542 & 4.2475 & - & - & - \\
\hline 1 & 1 & -2 & 4.1312 & 4.1196 & 4.1220 & 4.1078 & 4.0980 & 4.0939 & 4.0772 \\
\hline 1 & -1 & 2 & 3.9271 & 3.9224 & 3.9295 & 3.9061 & 3.8858 & - & - \\
\hline 0 & 0 & 3 & 3.4987 & 3.4794 & 3.4841 & 3.4673 & 3.4523 & 3.4449 & 3.4253 \\
\hline 3 & 0 & -1 & 3.3005 & 3.2936 & 3.2924 & 3.2892 & 3.2821 & 3.2821 & 3.2726 \\
\hline 2 & 0 & 2 & 3.2578 & 3.2508 & 3.2496 & - & - & - & - \\
\hline 1 & -2 & -1 & 3.1847 & 3.1729 & 3.1721 & 3.1681 & 3.1539 & 3.1523 & - \\
\hline 3 & 0 & -2 & 3.0551 & 3.0481 & 3.0481 & 3.0391 & 3.0317 & 3.0280 & - \\
\hline 3 & 0 & 1 & 2.9413 & 2.9340 & 2.9355 & 2.9271 & 2.9178 & 2.9141 & 2.9141 \\
\hline 3 & -2 & 1 & 2.8451 & 2.8244 & 2.8244 & 2.8234 & 2.8206 & 2.8113 & 2.8123 \\
\hline 3 & 0 & -3 & 2.6666 & 2.6600 & 2.6590 & 2.6521 & 2.6410 & 2.6382 & 2.6234 \\
\hline 2 & -2 & 3 & 2.5886 & 2.5930 & 2.5907 & 2.5910 & 2.5826 & 2.5734 & 2.5734 \\
\hline 0 & 2 & 2 & 2.5255 & 2.5252 & 2.5206 & 2.5132 & 2.5012 & 2.4799 & - \\
\hline 4 & -1 & 2 & 2.2438 & - & - & - & - & - & - \\
\hline 2 & 2 & 1 & 2.1780 & - & - & - & - & - & - \\
\hline 5 & -2 & 0 & 2.0433 & - & - & - & - & - & - \\
\hline 5 & -2 & 1 & 1.9848 & - & - & - & - & - & - \\
\hline 2 & -4 & 1 & 1.8154 & 1.8100 & 1.8120 & 1.8107 & 1.8052 & 1.8066 & 1.8066 \\
\hline
\end{tabular}


Table 3. High pressure x-ray diffraction results.

\begin{tabular}{|c|c|c|c|c|c|c|c|c|}
\hline P (GPa) & a (亡̊) & 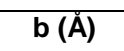 & c ( $(\dot{A})$ & $\alpha\left(^{\circ}\right)$ & $\beta\left(^{\circ}\right)$ & $Y\left(^{\circ}\right)$ & $V\left(\dot{A}^{3}\right)$ & Ref. \\
\hline ambient & "10.576(2) & $7.265(2)$ & "10.931(3) & (101.30(1) & 96.98(1) & $109.65(1)$ & 759.5 & [14] \\
\hline ambient & $10.52(1)$ & $7.25(1)$ & $10.92(2)$ & $101.4(1)$ & $96.9(1)$ & $109.4(1)$ & $755(2)$ & \multirow{7}{*}{$\begin{array}{c}\text { This study } \\
\text { (ME) }\end{array}$} \\
\hline $0.3(1)$ & $10.569(7)$ & $7.248(4)$ & $10.87(2)$ & $101.4(1)$ & $96.96(9)$ & $109.80(9)$ & $751(1)$ & \\
\hline $0.5(1)$ & $10.552(8)$ & $7.244(5)$ & $10.82(2)$ & $101.5(1)$ & $96.9(1)$ & 109.8(1) & $746(1)$ & \\
\hline $1.3(1)$ & $10.524(7)$ & $7.222(4)$ & $10.77(2)$ & $101.4(1)$ & $96.94(9)$ & $109.85(9)$ & $739(1)$ & \\
\hline $1.6(1)$ & $10.519(7)$ & $7.207(4)$ & $10.77(2)$ & $101.4(1)$ & $96.89(9)$ & $109.90(8)$ & $736(1)$ & \\
\hline $2.0(2)$ & $10.496(7)$ & $7.202(4)$ & $10.76(2)$ & $101.5(1)$ & $96.82(9)$ & $109.95(9)$ & $733(1)$ & \\
\hline $2.6(2)$ & $10.483(9)$ & $7.189(6)$ & $10.74(2)$ & $101.5(1)$ & $96.7(1)$ & $109.9(1)$ & $730(1)$ & \\
\hline $0.1(1)$ & $10.53(1)$ & $7.251(4)$ & $10.92(1)$ & $101.5(1)$ & $96.94(9)$ & $109.4(1)$ & $754(1)$ & \multirow{5}{*}{$\begin{array}{c}\text { This study } \\
\text { (SO) }\end{array}$} \\
\hline $0.5(1)$ & $10.52(1)$ & $7.247(5)$ & $10.88(1)$ & 101.6(1) & $96.8(1)$ & $109.5(1)$ & $749(1)$ & \\
\hline $0.9(1)$ & $10.49(1)$ & $7.241(4)$ & $10.83(1)$ & $101.7(1)$ & $96.77(9)$ & $109.5(1)$ & $743(1)$ & \\
\hline $1.3(1)$ & $10.48(1)$ & $7.237(4)$ & $10.80(1)$ & $101.7(1)$ & $96.66(9)$ & 109.6(2) & $740(1)$ & \\
\hline $2.0(2)$ & $10.46(4)$ & $7.22(1)$ & $10.73(4)$ & $101.7(5)$ & $96.5(2)$ & 109.6(4) & 733(4) & \\
\hline
\end{tabular}

Note: Standard deviations in parentheses.

Table 4 First-principles calculation results.

\begin{tabular}{|c|c|c|c|c|c|c|c|c|}
\hline$P(G P a)$ & a (文) & b (文) & c (六) & $\alpha\left(^{\circ}\right)$ & $\beta\left({ }^{\circ}\right)$ & $Y\left(^{\circ}\right)$ & $V\left(\dot{A}^{3}\right)$ & Ref. \\
\hline ambient & $10.576(2)$ & $7.265(2)$ & $10.931(3)$ & $101.30(1)$ & $96.98(1)$ & $109.65(1)$ & 759.5 & [14] \\
\hline-1.0 & 10.894 & 7.664 & 11.184 & 102.25 & 96.26 & 109.82 & 841.65 & \\
\hline 0.0 & 10.762 & 7.584 & 11.046 & 102.14 & 96.38 & 109.94 & 811.99 & \\
\hline 1.0 & 10.746 & 7.564 & 10.940 & 102.29 & 96.48 & 110.00 & 799.61 & \\
\hline 2.0 & 10.730 & 7.544 & 10.834 & 102.45 & 96.58 & 110.06 & 787.23 & $\begin{array}{c}\text { This study } \\
\text { (LDA) }\end{array}$ \\
\hline 3.0 & 10.663 & 7.510 & 10.807 & 102.42 & 96.62 & 110.04 & 776.93 & \\
\hline 4.0 & 10.627 & 7.491 & 10.766 & 102.45 & 96.75 & 110.04 & 768.90 & \\
\hline 5.0 & 10.619 & 7.485 & 10.663 & 102.60 & 97.23 & 110.07 & 758.07 & \\
\hline
\end{tabular}




\begin{tabular}{|c|c|c|c|c|c|c|c|c|}
\hline-1.0 & 10.673 & 7.327 & 11.006 & 100.90 & 97.76 & 109.33 & 779.20 & \\
\hline 0.0 & 10.626 & 7.292 & 10.912 & 100.98 & 97.71 & 109.35 & 765.22 & \\
\hline 1.0 & 10.579 & 7.261 & 10.826 & 101.00 & 97.81 & 109.34 & 752.22 & \\
\hline 2.0 & 10.535 & 7.234 & 10.742 & 101.03 & 97.85 & 109.36 & 740.12 & $\begin{array}{c}\text { This study } \\
\text { (GGA) }\end{array}$ \\
\hline 3.0 & 10.506 & 7.210 & 10.628 & 101.05 & 97.95 & 109.32 & 727.70 & \\
\hline 4.0 & 10.472 & 7.187 & 10.542 & 101.08 & 98.02 & 109.31 & 716.95 & \\
\hline 5.0 & 10.440 & 7.166 & 10.462 & 101.14 & 98.00 & 109.33 & 707.04 & \\
\hline
\end{tabular}

Table 5. Calculated elastic coefficients.

\begin{tabular}{|c|c|c|c|c|c|c|c|c|c|c|c|c|c|}
\hline \multicolumn{14}{|c|}{ Jennite computation } \\
\hline & \multicolumn{2}{|c|}{ LDA } & \multicolumn{2}{|c|}{ GGA } & \multirow{2}{*}{$\begin{array}{c}\text { GGA [16] } \\
X / / a\end{array}$} & \multirow{2}{*}{$\begin{array}{l}\text { LDA [17] } \\
\text { n.d. }\end{array}$} & & \multicolumn{2}{|c|}{ LDA } & \multicolumn{2}{|c|}{ GGA } & \multirow{2}{*}{$\begin{array}{c}\text { GGA [16] } \\
x / / a\end{array}$} & \multirow{2}{*}{$\begin{array}{c}\text { LDA [17] } \\
\text { n.d. }\end{array}$} \\
\hline & $\mathrm{Z} / / \mathrm{c}$ & $\mathbf{X} / \mathbf{a}$ & $\mathrm{Z} / / \mathrm{c}$ & $\mathbf{X} / \mathbf{a}$ & & & & $\mathrm{Z} / / \mathrm{c}$ & $\mathbf{X} / \mathbf{a}$ & $\mathrm{Z} / / \mathrm{c}$ & $\mathbf{X} / \mathbf{a}$ & & \\
\hline c11 & 99.9 & 100.0 & 1111.7 & 1110.4 & 100.1 & 107.09 & K_Voigt & 63.1 & 64.5 & 61.2 & 60.8 & 36.8 & - \\
\hline c12 & 44.5 & 49.0 & 41.6 & 40.9 & 26.9 & 40.52 & K_Reuss & 59.8 & 61.4 & 57.9 & 58.2 & 26.8 & - \\
\hline c13 & 45.9 & 46.6 & 39.2 & 41.1 & 32.0 & 37.30 & K_RVH & 61.5 & 63.0 & 59.6 & 59.5 & 31.8 & 62.7 \\
\hline c14 & -8.4 & -6.7 & 0.0 & 1.5 & 1.3 & - & G_Voigt & 30.1 & 29.6 & 33.8 & 33.9 & 23.3 & - \\
\hline c15 & 4.4 & 4.9 & 2.6 & 5.1 & 1.5 & - & G_Reuss & 26.8 & 26.2 & 31.5 & 31.9 & 20.6 & - \\
\hline c16 & -1.9 & -3.0 & 4.2 & 1.9 & 3.3 & - & G_RVH & 28.4 & 27.9 & 32.7 & 32.9 & 22.0 & 35.0 \\
\hline c22 & 128.2 & 127.5 & 130.1 & 126.9 & 45.7 & 122.97 & E_RVH & 73.9 & 72.9 & 82.8 & 83.3 & 53.6 & 88.6 \\
\hline c23 & 39.6 & 41.7 & 33.6 & 33.9 & 4.4 & 35.39 & n_RVH & 0.3 & 0.3 & 0.3 & 0.3 & 0.2 & 0.3 \\
\hline c24 & -4.2 & 3.0 & -1.9 & 1.2 & 7.4 & - & & & & & & & \\
\hline c25 & -3.1 & -4.9 & -2.3 & -5.7 & -6.2 & - & & & & & & & \\
\hline c26 & -10.4 & -6.9 & -9.8 & -9.6 & -3.2 & - & & & & & & & \\
\hline c33 & 80.3 & 78.8 & 80.3 & 78.4 & 59.2 & 73.45 & & & & & & & \\
\hline c34 & -0.5 & 0.3 & -5.0 & -2.6 & -1.3 & - & & & & & & & \\
\hline c35 & -8.2 & -6.0 & -6.4 & -1.5 & 1.4 & - & & & & & & & \\
\hline c36 & 0.2 & -1.4 & 3.5 & 3.1 & 0.1 & - & & & & & & & \\
\hline c44 & 24.0 & 23.3 & 32.0 & 32.5 & 22.0 & 25.11 & & & & & & & \\
\hline
\end{tabular}




$\begin{array}{ccccccc}\text { c45 } & -0.7 & -3.2 & 0.5 & -0.2 & -1.7 & - \\ \text { c46 } & -4.2 & \mathbf{1 . 8} & -1.5 & -0.3 & -1.6 & - \\ \text { c55 } & 31.7 & \mathbf{2 7 . 0} & 27.7 & 30.3 & 21.0 & 23.38 \\ \text { c56 } & -1.6 & -\mathbf{0 . 6} & 1.7 & 1.2 & 2.7 & - \\ \text { c66 } & 35.1 & \mathbf{4 1 . 1} & 40.2 & 40.2 & 26.6 & 39.45\end{array}$

Note: Most reliable theoretical results are in bold.

Table 6. Summary of the mechanical properties of jennite.

\begin{tabular}{|c|c|c|c|c|c|c|c|c|}
\hline & & \multicolumn{2}{|c|}{$\begin{array}{l}\text { Experimental } \\
\text { study }\end{array}$} & \multicolumn{5}{|c|}{ Theoretical study } \\
\hline & & ME & SL & LDA & GGA & GGA [16] & MD [18] & LDA [17] \\
\hline \multirow{3}{*}{$3^{\text {rd }}$ EoS } & $\overline{\mathrm{K}_{0}(\mathrm{GPa})}$ & 45(5) & (61(4) & 62(7) & $\begin{array}{l}57(1) \\
\end{array}$ & - & - & - \\
\hline & $\mathrm{K}_{0}{ }^{\prime}$ & 42.3 & 9.9 & 5.1 & 2.4 & - & - & - \\
\hline & $V_{0}\left(\AA^{3}\right)$ & $754(2)$ & $755(1)$ & 812 & 765.2 & - & - & - \\
\hline \multirow{2}{*}{$2^{\text {nd }}$ EoS } & $\mathrm{K}_{0}(\mathrm{GPa})$ & $68(5)$ & $64(2)$ & $63(1)$ & $54.2(8)$ & - & - & - \\
\hline & $V_{0}\left(\AA^{3}\right)$ & $754(2)$ & $755(1)$ & 812 & 765.2 & 775 & - & 764.5 \\
\hline \multirow{4}{*}{ RVH } & K (GPa) & - & - & $63(2)$ & $60(1)$ & $32(5)$ & 43 & 62.7 \\
\hline & G (GPa) & - & - & $28(2)$ & $33(1)$ & $22(1)$ & 26 & 35.0 \\
\hline & $E(G P a)$ & - & - & 72.9 & 83.3 & 53.6 & 66 & 88.6 \\
\hline & $\eta$ & - & - & 0.3 & 0.3 & 0.2 & 0.2 & 0.3 \\
\hline
\end{tabular}

Note: Most reliable theoretical results are in bold.

Table 7. Mean squared residuals of length and angle of lattice parameters and volume: Comparison between LDA and GGA exchange-correlation functionals.

\begin{tabular}{c|c|c}
\hline \multirow{2}{*}{ Parameter } & \multicolumn{2}{|c}{ Mean squared residual $\left(\times 10^{-2}\right)$} \\
\cline { 2 - 3 } & LDA & GGA \\
\hline \hline Lengths & 5.496 & 11.655 \\
\hline
\end{tabular}




\begin{tabular}{c|c|c} 
Angles & 5.993 & 6.762 \\
Volume & 4.846 & 12.256 \\
\hline
\end{tabular}


Figure 1. Jennite crystal collected from Zeilberg, Bavaria, Germany.

Figure 2. X-ray diffraction patterns of jennite $(\lambda=0.6199 \AA)$ with alcohol mixture (Methanol:Ethanol $=$ 4:1). Bottom peaks indicate reference peak positions from [14].

Figure 3. X-ray diffraction patterns of jennite $(\lambda=0.4959 \AA)$ with silicone oil. Bottom peaks indicate reference peak positions from [14].

Figure 4. Variation of (a) axial and (b) angular lattice parameters of jennite under pressure. Closed and open symbols correspond to refined parameters measured with alcohol mixture (Methanol:Ethanol $=4: 1$ ) and silicone oil, respectively. Red and black lines indicate DFT simulation result using LDA and GGA, respectively.

Figure 5. (a) Refined unit cell volumes of jennite under pressure. (b) F-f plot of jennite. The $2^{\text {nd }}$ order BM EoS fittings give the bulk modulus of 68(5) GPa and 64(2) GPa with alcohol mixture and silicone oil, respectively.

Figure 6. Geometrically optimized jennite projected along [010]. Silicate chains, calcium octahedra, and oxygen atoms are shown as dark blue, light blue tetrahedra, and red spheres, respectively. Hydroxyl groups and hydrogen atoms in water molecules are denoted with black sticks.

Figure 7. Comparison of volumetric compressibilities of jennite from high pressure experiments (box), previous GGA (dotted line) simulation result [16], and current LDA (red line) and GGA (black line) simulations.

Figure 8. Directional Young's modulus of jennite computed from (a) LDA and (b) GGA functionals. Elastic constants calculated in the conventional orthogonal coordinate system $(\vec{Z}\|\vec{c}, \vec{Y}\| \vec{c} \times \vec{a}$, and $\vec{X} \| \vec{Y} \times \vec{Z}$ ) are used. Scale bars indicate Young's modulus in GPa. 
Figure 9. Q-Q plot analysis of residuals: total residuals of length $(a, b$, and $c)$ and angles $(\alpha, \beta$, and $\gamma)$ obtained from HPXRD and DFT calculations. 


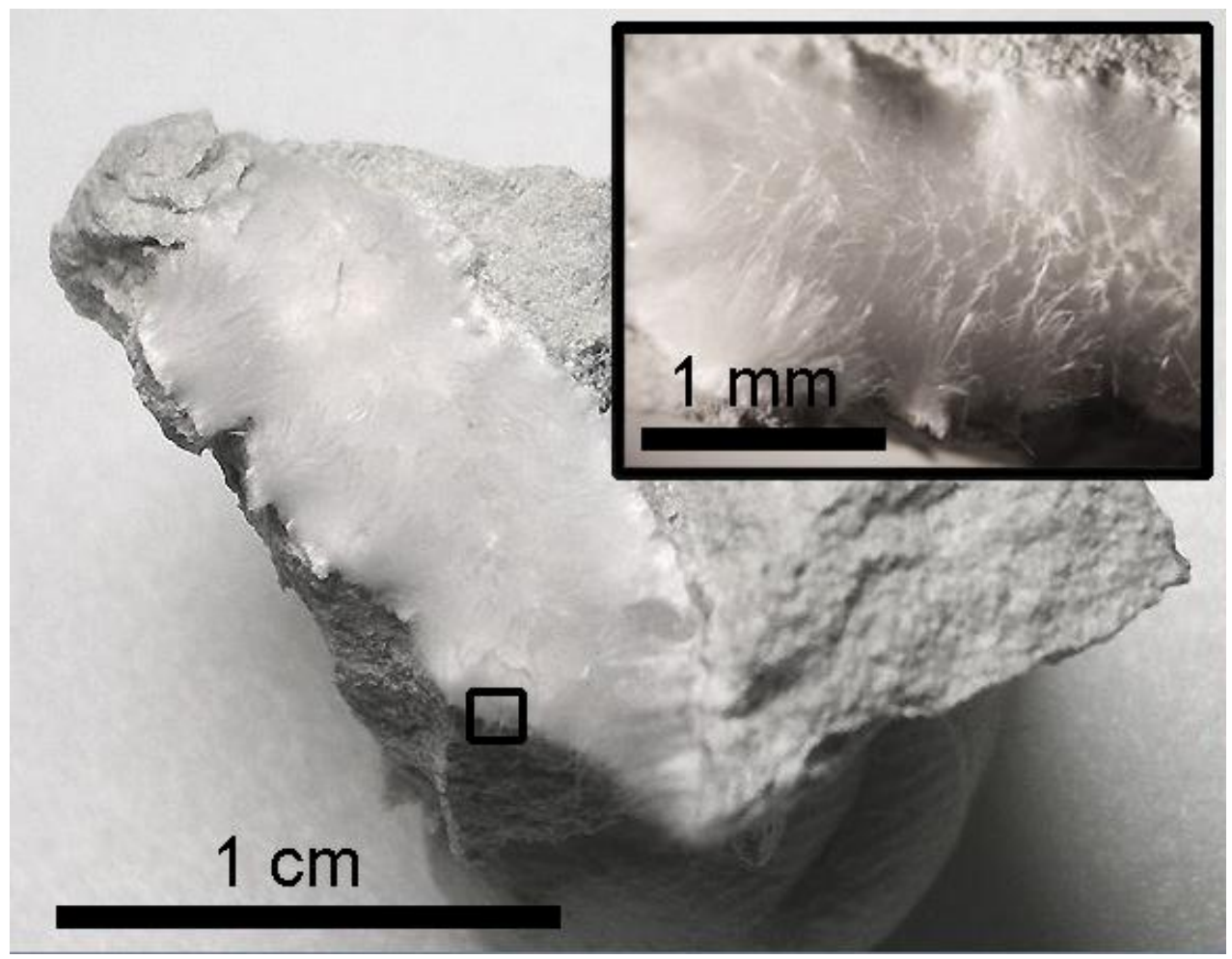

Figure 1 


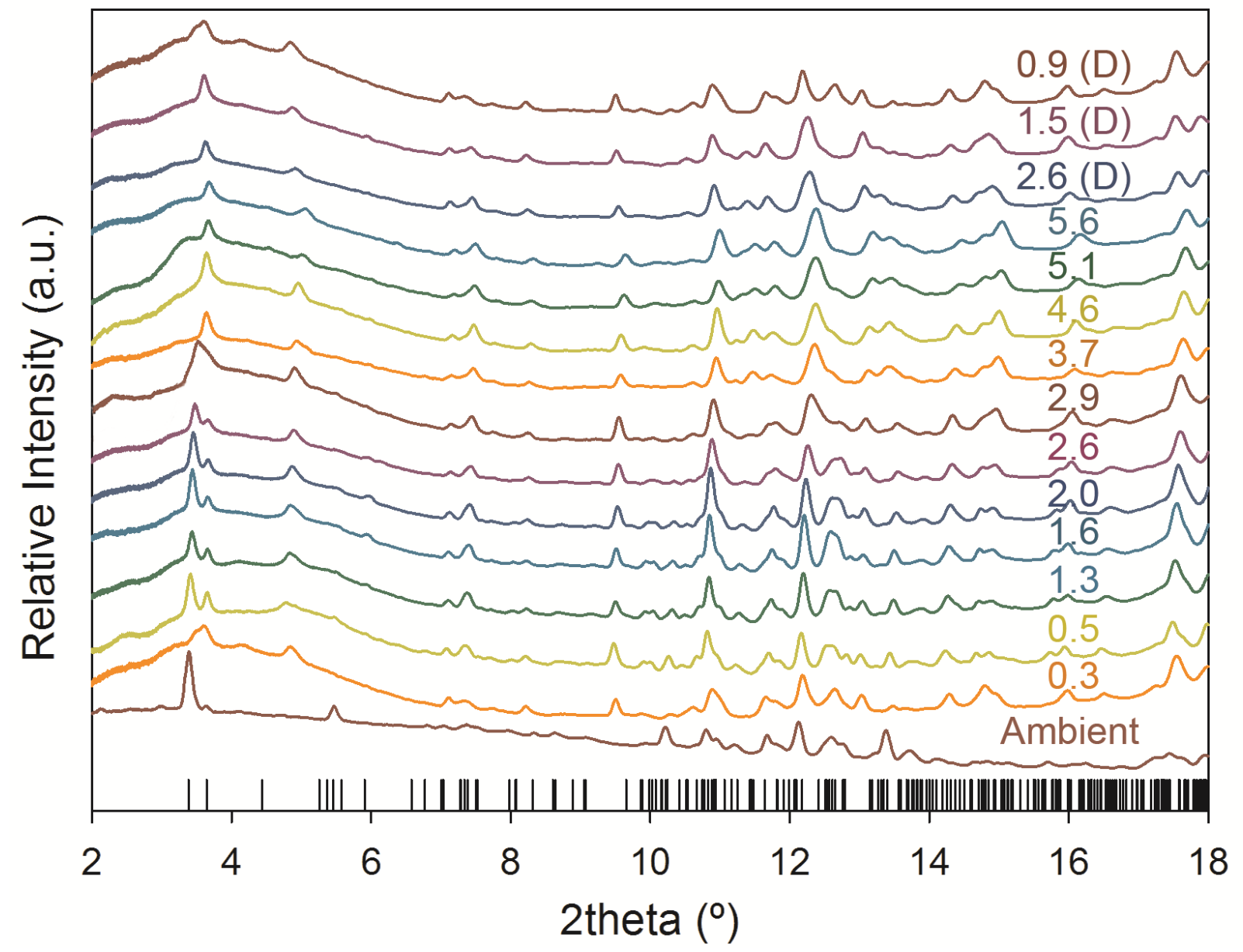

Figure 2 


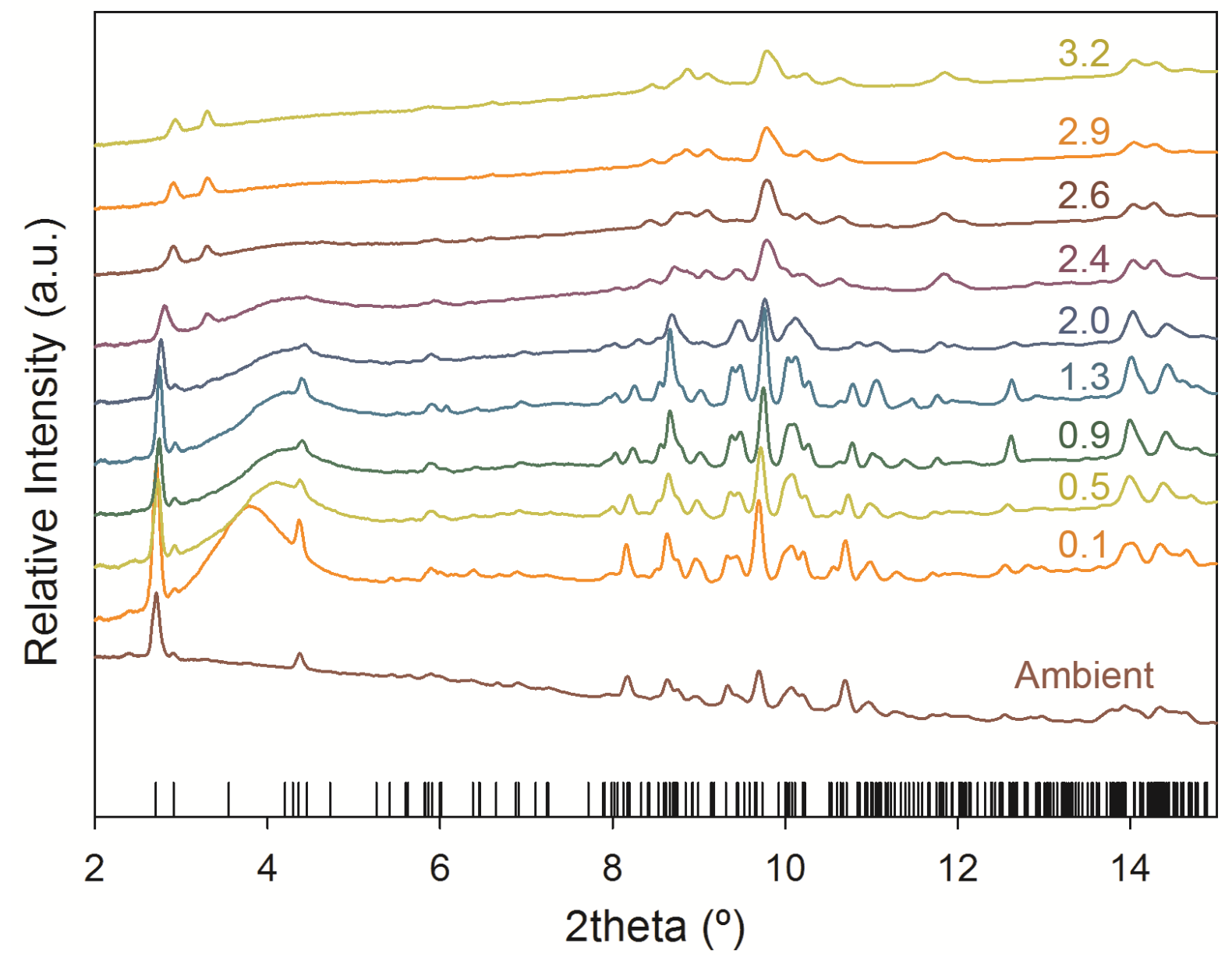

Figure 3 


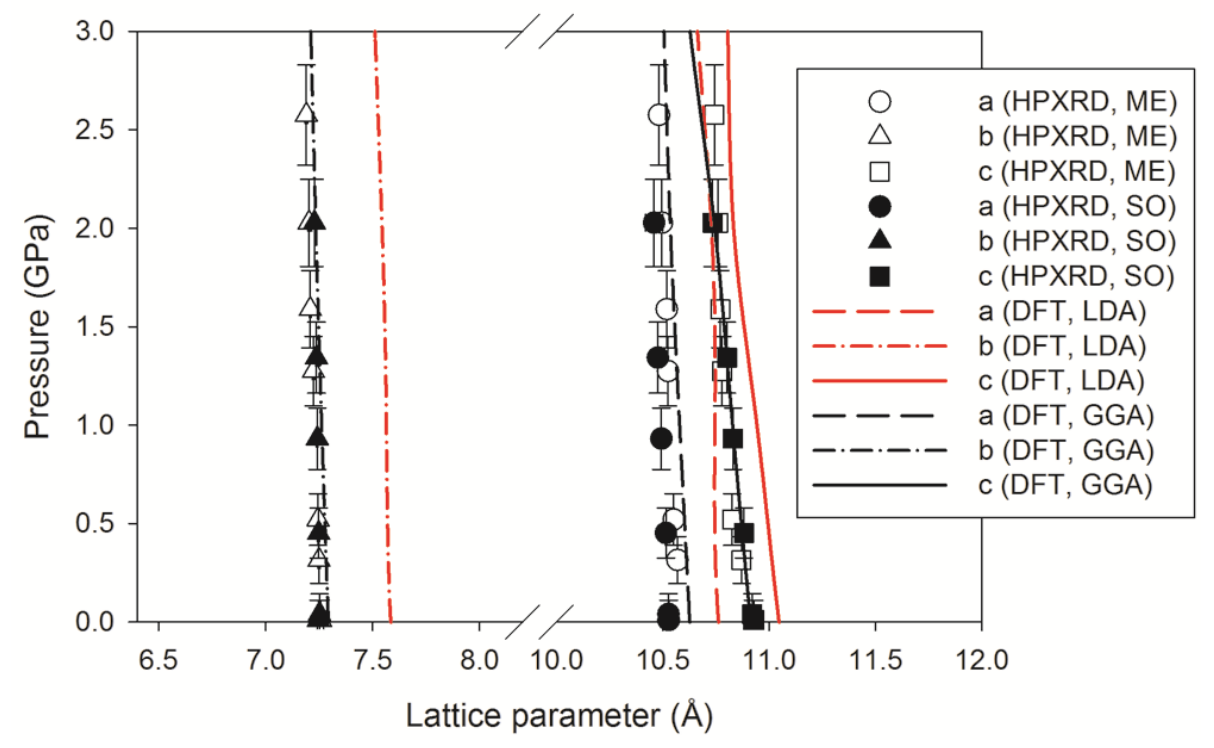

Figure 4a 


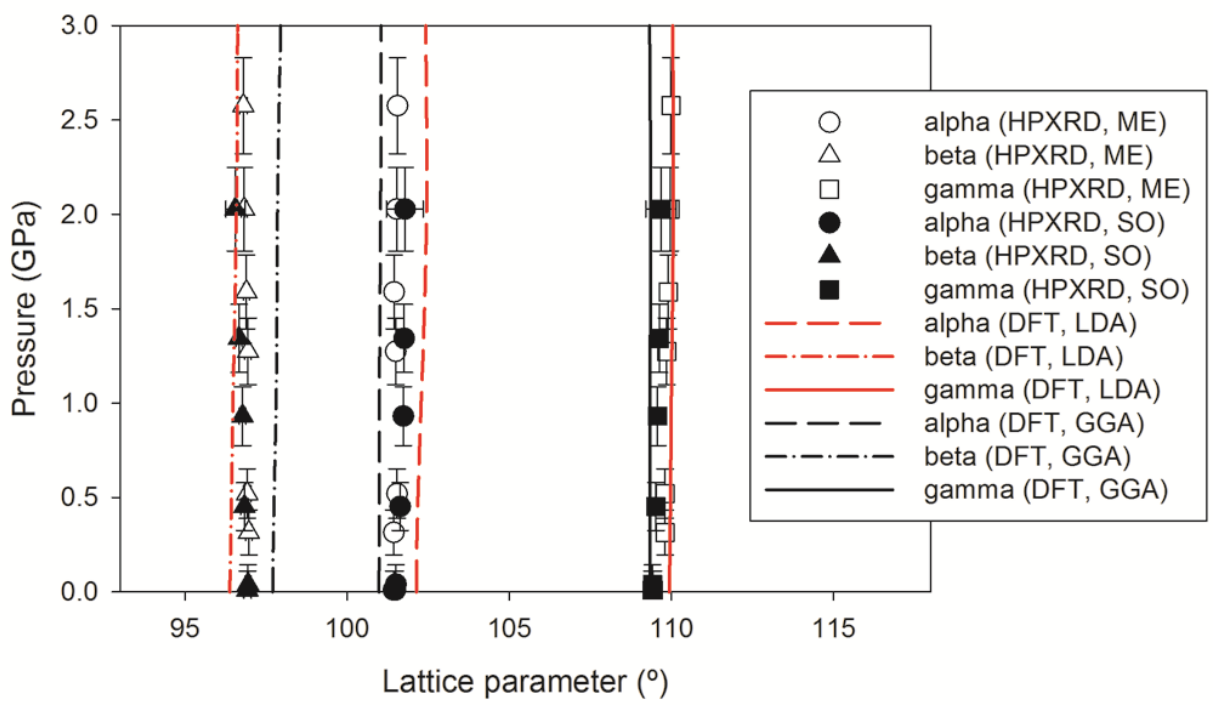

Figure $4 b$ 


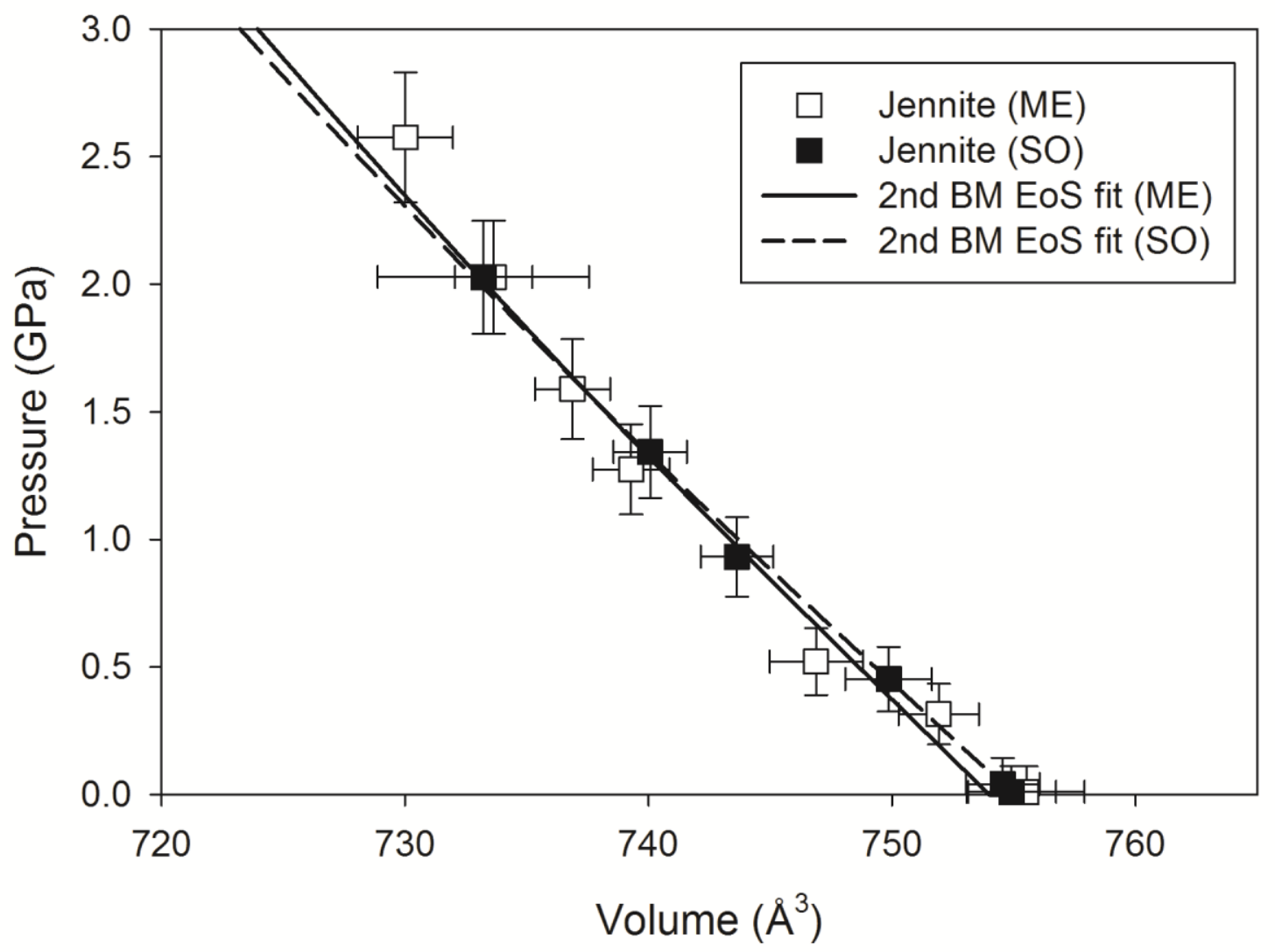

Figure 5a 


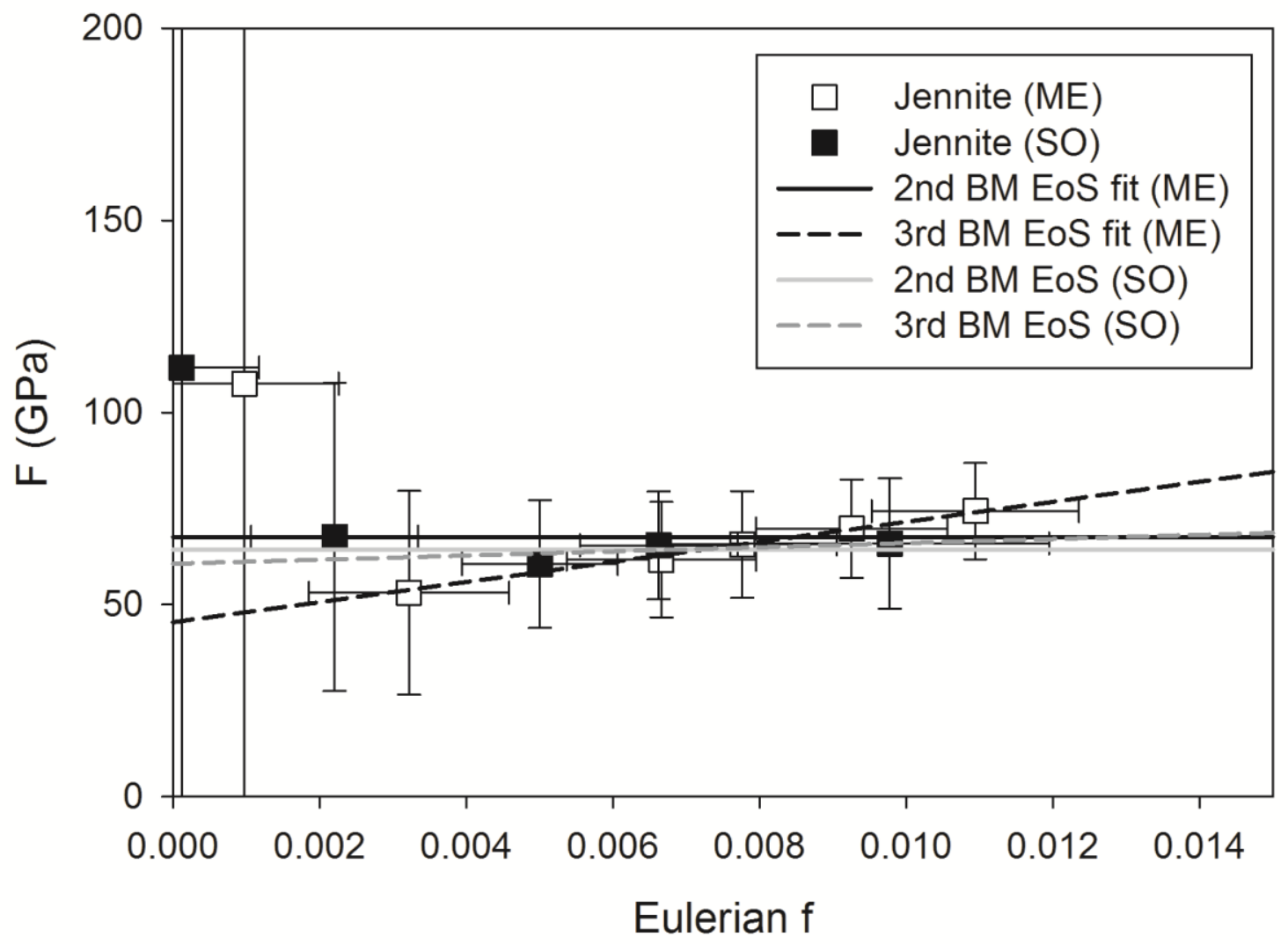

Figure $5 b$ 
0

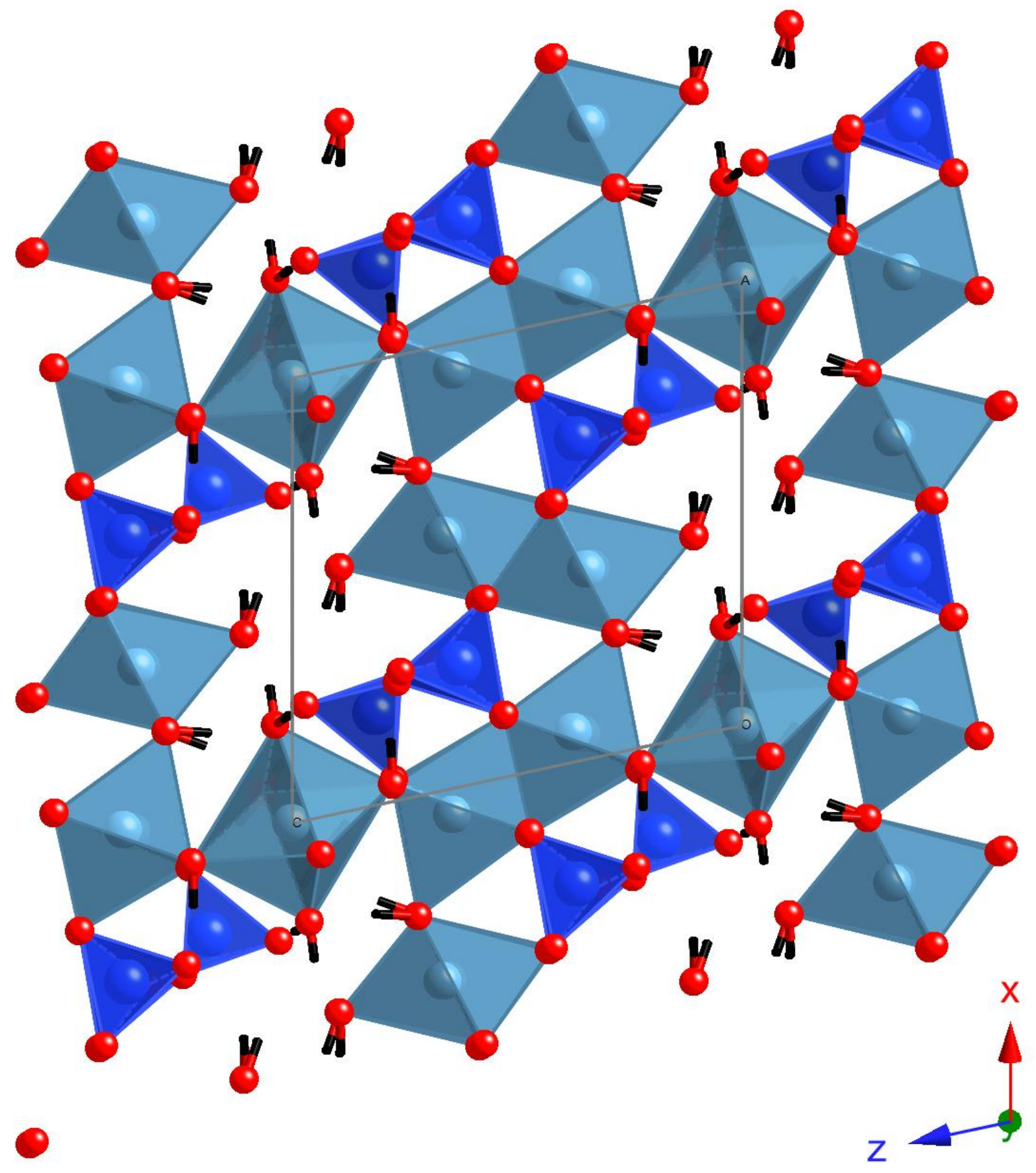

Figure 6 


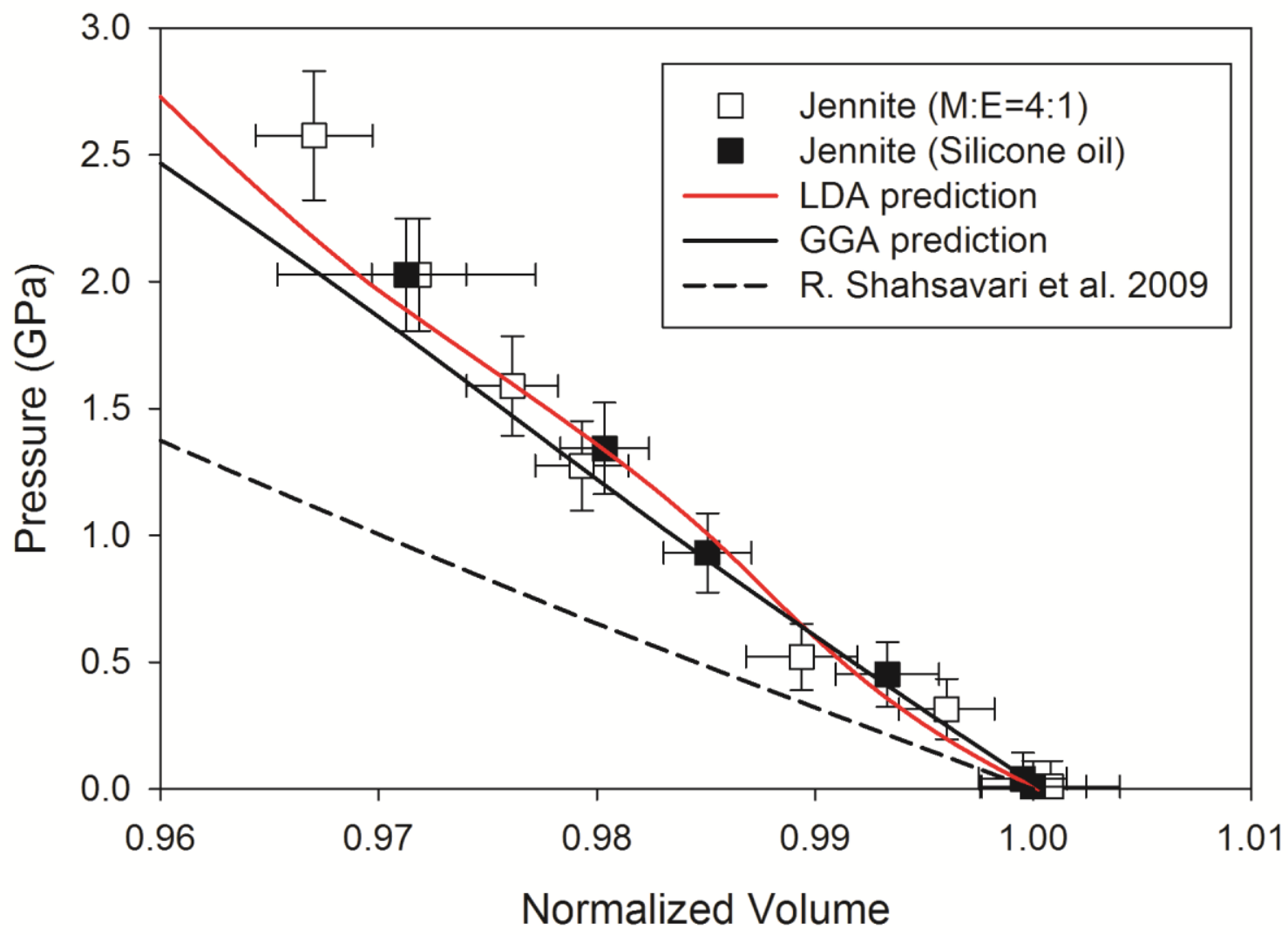

Figure 7 


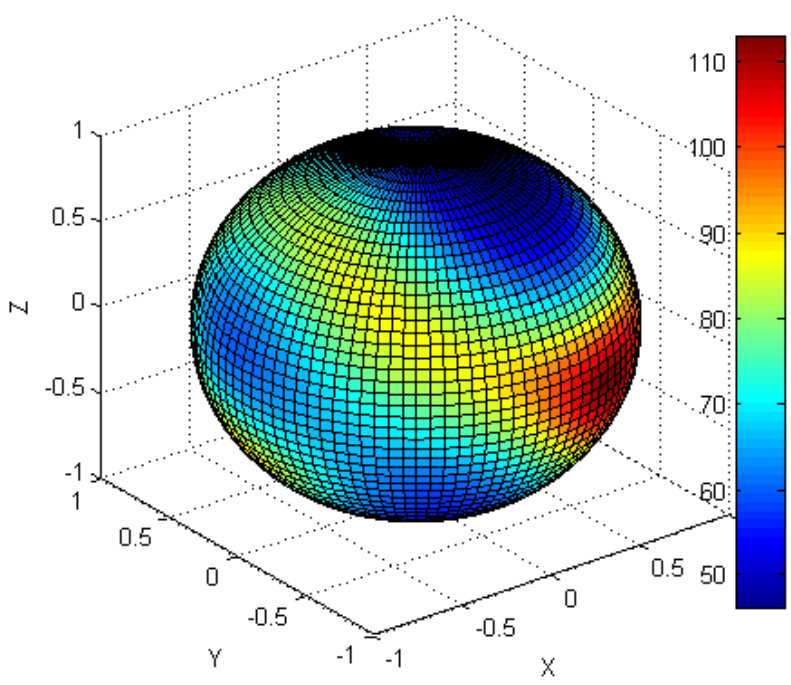

(a) LDA

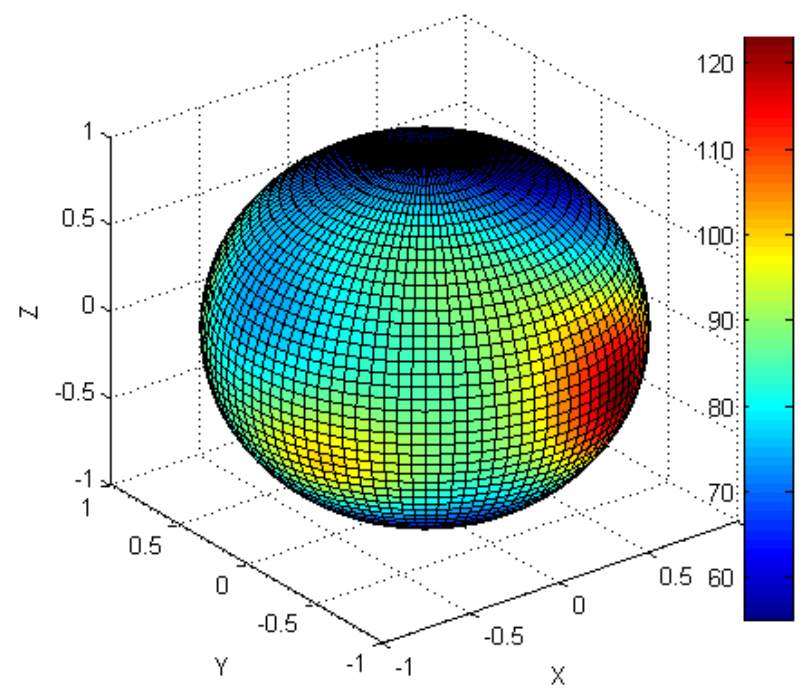

(b) GGA

Figure 8 

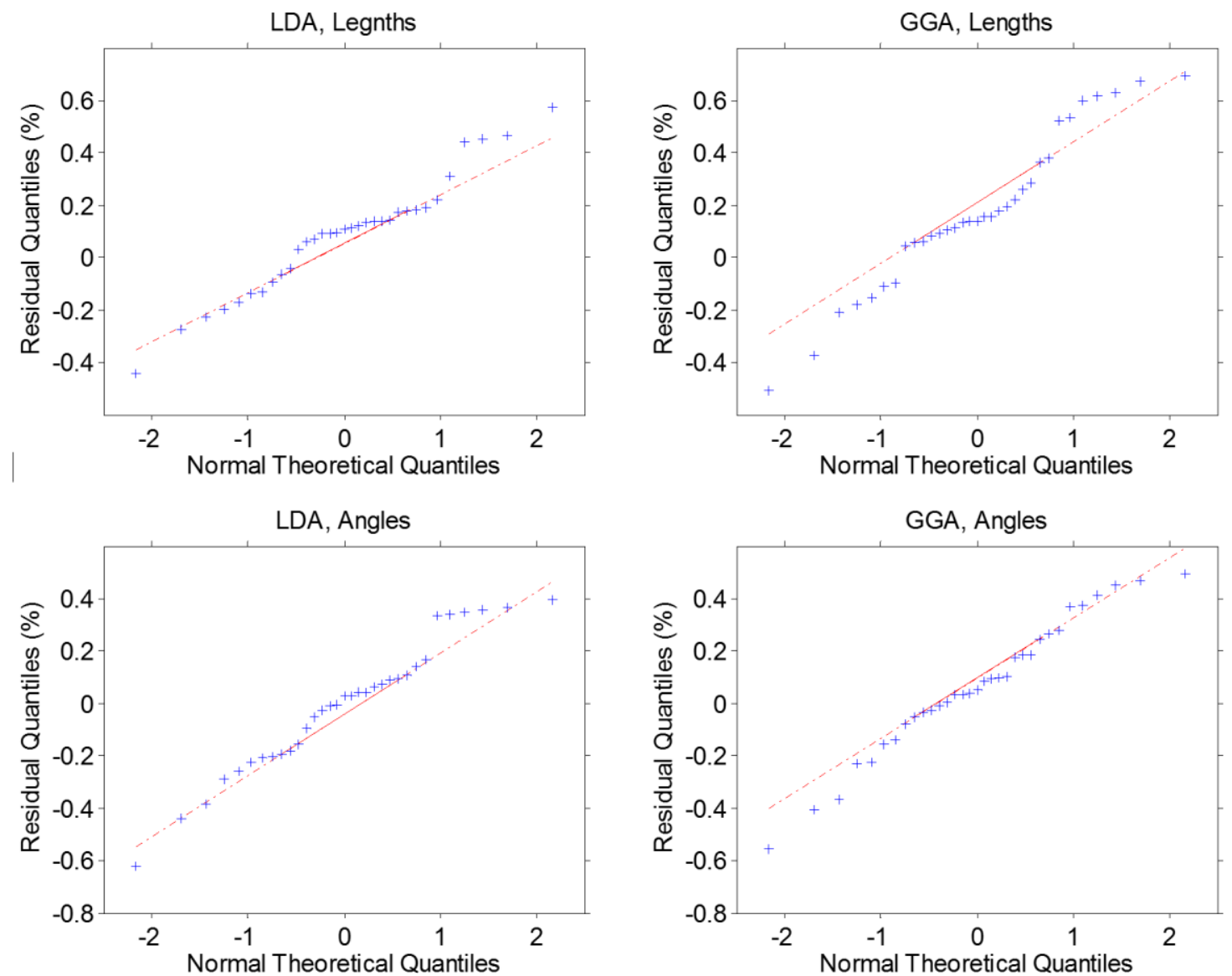

Figure 9 